\title{
The impact of naltrexone and morphine tolerance on mild shock-induced hypoalgesia
}

\author{
JAMES W. GRAU, MANDY K. BILES, and PAUL A. ILLICH \\ Texas A \& M University, College Station, Texas
}

\begin{abstract}
We have previously shown that exposure to mild shock elicits a strong hypoalgesia on the tailflick test in rats. The present studies explored the role of endogenous opioids in producing this hypoalgesia. Experiment 1 evaluated the impact of five doses of naltrexone $(0,0.44,1.75,7$, and $28 \mathrm{mg} / \mathrm{kg}$ ); Experiment 2 looked at the impact of morphine tolerance. Both a low dose of naltrexone $(1.75 \mathrm{mg} / \mathrm{kg})$ and morphine tolerance attenuated the hypoalgesia observed 6-10 min after mild shock. By contrast, neither morphine tolerance nor a high dose of naltrexone $(28 \mathrm{mg} / \mathrm{kg})$ had a significant impact on the hypoalgesia observed 2 min after shock. These findings suggest that mild shock elicits both a transient nonopioid and a long-lasting opioid hypoalgesia.
\end{abstract}

We have previously shown that mild shock (three 0.75 sec, 1.0-mA shocks) elicits a strong hypoalgesia in rats, as measured by the tailflick test (Grau, 1984, 1987a, 1987b). We have also demonstrated that a high dose of either naloxone or naltrexone attenuates the hypoalgesia observed 6-10 min after shock but not the hypoalgesia observed just 2 min after shock (Grau, 1984, 1987b). On the basis of these findings, we have argued that mild shock elicits both a transient nonopioid and a long-lasting opioid hypoalgesia. This pattern of results has provided a cornerstone to a theory proposed by Grau $(1986,1987 \mathrm{~b})$ and the foundation for considerable empirical work (e.g., Grau, 1987a, 1987b; Grau, Illich, Chen, \& Meagher, 1991; Meagher, Grau, \& King, 1989, 1990). It is, therefore, quite troubling that serious questions can be raised regarding our basic claim that both nonopioid and opioid mechanisms play a role in mild shock-induced hypoalgesia. The primary problem is that we have relied upon a single manipulation, reversal by a high dose of opioid antagonist (e.g., $28 \mathrm{mg} / \mathrm{kg}$ of naltrexone), to infer opioid mediation. Such a high dose of antagonist may have its impact by distupting other neurochemical systems (Sawynok, Pinsky, \& LaBella, 1979). To refute this criticism, evidence is needed that a low dose of an opioid antagonist, and other manipulations that undermine opioid function (e.g., opioid tolerance), have a similar impact.

Unfortunately, this issue cannot be addressed by simply referring to other work in the field. The problem here is that there are a number of distinct forms of opioid and nonopioid hypoalgesia, many of which appear to be paradigm-specific. For example, consider the opioid and nonopioid hypoalgesia observed after relatively long (e.g.,

\footnotetext{
Work on this manuscript was supported by grants from the National Institute of Drug Abuse Grant (DA04259-01 and DA05846-01) and National Science Foundation (BNS-8819981) to J. W. Grau. Correspondence concerning this article should be addressed to James W. Grau, Department of Psychology, Texas A \& M University, College Station, TX 77843 .
}

greater than $60 \mathrm{sec}$ ) and intense (e.g., $1.6 \mathrm{~mA}$ ) continuous shocks (Terman, Shavit, Lewis, Cannon, \& Liebeskind, 1984; Watkins \& Mayer, 1982). In these paradigms, it is clear that a low dose of opioid antagonist blocks the putative opioid, but not the nonopioid, hypoalgesic response (Terman et al., 1984; Terman, Lewis, \& Liebeskind, 1986; Watkins, Cobelli, Faris, Aceto, \& Mayer, 1982). However, the hypoalgesia observed in these paradigms, unlike the hypoalgesia we observe after mild shock, is not affected by manipulations that disrupt forebrain functioning (e.g., administration of pentobarbital, decerebration, or lesions of the frontal cortex [Meagher, Grau, \& King, 1989, 1990; Terman et al., 1984; Watkins, Kinscheck, \& Mayer, 1983]). In a paradigm that appears more similar to our own, Maier (1989) has shown that a single 5-sec tailshock generates a hypoalgesia that is blocked by $7 \mathrm{mg} / \mathrm{kg}$ of naltrexone and that five 5-sec tailshocks elicit both a transient nonopioid and a long-lasting opioid hypoalgesia. But, again, these hypoalgesic responses are not eliminated by a manipulation that disrupts forebrain functioning (Maier, 1989). Although this has not been explicitly tested, it does seem likely that the opioid hypoalgesia Fanselow (1984) observes after mild gridshock on the formalin test depends on forebrain systems (Maier, 1989; Meagher et al., 1989, 1990). However, here, too, it is unclear whether we can generalize from this paradigm to our own, for evidence suggests the pain associated with the formalin test can change the form of the observed hypoalgesia from nonopioid to opioid (Maier, Ryan, \& Kurtz, 1984). Moreover, the affective impact of shock probably varies, depending on whether it is delivered through grids or tail electrodes and whether or not the animal is immobilized during testing. In fact, a comparison of the shock parameters used by different researchers suggests that much less severe shocks are needed to engage hypoalgesic systems when the shock is presented through tail electrodes and the subjects are restrained (cf. Maier, 1989; Meagher et al., 1990; Terman et al., 1984). Given this, it seems likely 
that our tailshock/restraint procedure might be more comparable to the more intense gridshock paradigm that was found by Fanselow (1984) to elicit a strong nonopioid hypoalgesia. What these observations suggest is that we cannot answer the question of whether our mild shock paradigm engages an opioid hypoalgesia by simply referring to other work.

The present experiments are designed to establish the form of the hypoalgesia observed in our mild shock paradigm. Experiment 1 looks at the impact of naltrexone over a wide range of doses; Experiment 2 evaluates the effect of morphine tolerance.

\section{EXPERIMENT 1 Impact of Naltrexone}

As mentioned above, others have noted that the high dose of opioid antagonist used by Grau (1987b) could have disrupted other neurochemical systems. For example, it may have disrupted GABAergic function (Sawynok et al., 1979). This is quite problematic, since evidence exists that GABA may play a role in environmentally induced hypoalgesia (Drugan, McIntyre, Alpern, \& Maier, 1985; Drugan, Ryan, Minor, \& Maier, 1984). Thus, our "opioid" hypoalgesia might actually be mediated by GABAergic systems. To rule out this possibility, we need to demonstrate that a much lower dose of naltrexone can block the hypoalgesia observed 6-10 min after shock. One can also question our claim that the hypoalgesia observed 2 min after shock is nonopioid in nature (Maier, 1989; Matzel \& Miller, 1989). The problem here is that we have always injected the naltrexone immediately before the subjects are placed in the test apparatus. Given this, it is possible that naltrexone failed to reverse the hypoalgesia observed 2 min after shock simply because it did not have enough time to act. To rule out this possibility, we need to demonstrate that a similar pattern of results is observed when the drug is given additional time to act. The present experiment is designed to address both of these problems. First, we test the impact of naltrexone over a wide range of doses $(0.0,0.44,1.75,7.0$, and $28 \mathrm{mg} / \mathrm{kg})$. Second, we allow the drug an additional $15 \mathrm{~min}$ to exert its effect.

\section{Method}

Subjects. The subjects were 100 Sprague-Dawley rats obtained from Harlan (Houston, Texas). The rats were 100-120 days old and weighed between 420 and $480 \mathrm{~g}$. They were maintained on 12:12-h light:dark cycle. Testing was conducted during the last $3 \mathrm{~h}$ of the light cycle. The subjects were individually housed and had food and water continuously available.

Apparatus. During behavioral testing, the rats were restrained in one of two Plexiglas tubes. A 660-V transformer was used to produce a constant-current $1.0-\mathrm{mA}$ shock. The shock was administered through tail electrodes that were constructed from a modified fuse clip. The electrode was taped to the rat's tail, approximately $15 \mathrm{~cm}$ behind the rear of the tubes. Pain responsivity was assessed with a radiant-heat tailflick device. A detailed description of this device, as well as other details of the apparatus, can be found elsewhere (Grau, 1987a, 1987b).
Procedure. The experiment employed a 5 (drug condition) $\times$ 2 (shock condition) factorial design. Subjects received an i.p. injection of either $0.0,0.44,1.75,7$, or $28 \mathrm{mg} / \mathrm{kg}$ of naltrexone dissolved in saline. Fifteen minutes later, the subjects were placed in the unilluminated restraining tubes and allowed to acclimate themselves for $15 \mathrm{~min}$. The subjects then received four tailflick tests at 2-min intervals. An 8-sec cutoff was used to prevent tissue damage. The last three tests were used to compute the subject's baseline level of pain reactivity.

After the last tailflick test, the electrodes were attached with adhesive tape. Half of the subjects in each drug condition were then given three inescapable, unsignaled $1.0-\mathrm{mA}, 0.75$-sec shocks at 20sec intervals. The other subjects were treated the same, except that shock was withheld. About $1 \mathrm{~min}$ after the last shock, or an equivalent period of restraint, the rat's tail was untaped. A minute later, five tailflick tests were conducted at 2 -min intervals.

Statistics. On the basis of prior work, it was hypothesized that naltrexone would have little impact at 2 min after shock and would attenuate the analgesia observed 6-10 min after shock. To test this hypothesis, two separate ANOVAs were conducted: one on the data obtained from the 2-min test point; another on the 6-10-min means. Duncan's multiple range test $(p<.05)$ was used to make post hoc comparisons.

\section{Results and Discussion}

The impact of naltrexone on baseline levels of pain reactivity is depicted in the top panel of Figure 1. Although the drug appears to have produced a slight hyperalgesia, this effect did not approach statistical significance (all $F$ s $\langle 1.0, p\rangle .05$ ).

The impact of naltrexone on shock-induced hypoalgesia is depicted in the lower two panels of Figure 1. At both 2 and 6-10 min after testing, it is clear that shock induced a strong hypoalgesia in the saline controls. At 2 min after shock (middle panel), naltrexone appears to have had relatively little impact on the hypoalgesia. By contrast, at 6-10 min after shock (bottom panel), the hypoalgesia was attenuated by the three largest doses of naltrexone $(1.75,7$, and $28 \mathrm{mg} / \mathrm{kg})$. The lowest dose $(0.44 \mathrm{mg} / \mathrm{kg})$ had no impact.

Statistical analyses confirmed these impressions. An analysis of the data from the 2-min test point revealed a significant shock effect $[F(1,90)=61.67, p<.001]$. However, neither the main effect of drug treatment $[F(4,90)=0.85, p>.05]$ nor its interaction with shock treatment $[F(4,90)=0.89, p>.05]$ approached statistical significance. Post hoc comparisons confirmed that the five shocked groups were hypoalgesic relative to the five unshocked groups $(p<.05)$. None of the differences between the five shocked groups approached statistical significance $(p>.05)$. Similarly, the unshocked groups did not differ significantly.

An analysis of the 6-10-min means revealed shock had a significant impact $[F(1,90)=31.63, p<.001]$. Although the main effect of drug was not significant $[F(4,90)=2.10, p<.10]$, trend analysis revealed a significant linear component $[F(1,90)=5.44, p<.05]$. The quadratic, cubic, and quartic components did not approach statistical significance (all $F \mathrm{~s}<2.16, p>.05$ ). Similarly, although the interaction between shock and drug 

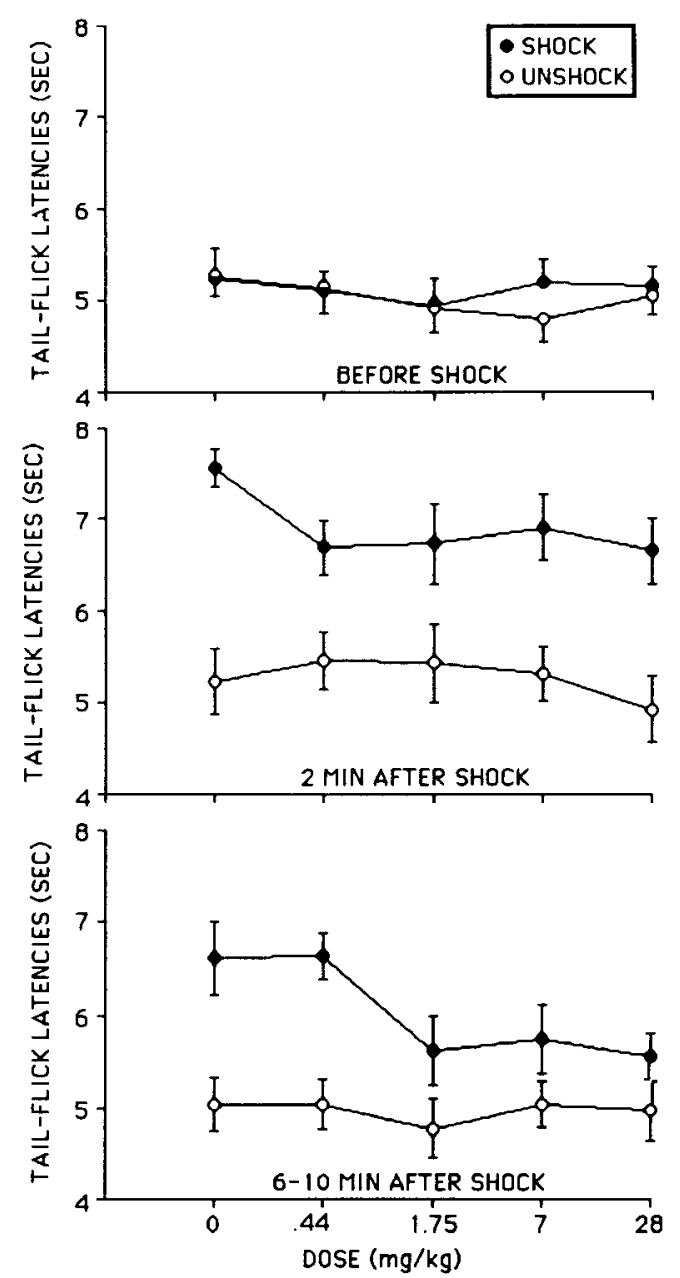

Figure 1. The mean levels of pain reactivity observed prior to shock (top panel), 2 min after shock (middle panel), and 6-10 min after shock (bottom panel). (The filled and unfilled circles depict the data from the shocked and unshocked groups, respectively. The error bars indicate the standard error of the mean [SEM].)

treatment was not significant $[F(1,90)=1.34, p>.05]$, its linear component was significant $[F(1,90)=4.56$, $p<.05]$. Post hoc comparisons revealed that the shocked groups that received saline or $0.44 \mathrm{mg} / \mathrm{kg}$ of naltrexone were hypoalgesic relative to all other groups. No other differences were significant.

Thus, as in prior studies (Grau, 1984, 1987b), naltrexone had no impact on the hypoalgesia observed 2 min after shock. Importantly, this was true even though the drug was given an additional $15 \mathrm{~min}$ to exert its action. This rules out the possibility that naltrexone failed to have a significant impact on the hypoalgesia observed $2 \mathrm{~min}$ after shock simply because it did not have enough time to act. Thus, this hypoalgesia appears to be mediated by a nonopioid system(s). In contrast, a low dose of naltrexone $(1.75 \mathrm{mg} / \mathrm{kg})$ blocked the hypoalgesia observed 6-10 min after shock. Since such a low dose of naltrexone should have a minimal impact on nonopioid systems (e.g., GABAergic), this finding suggests the hypoalgesia observed 6-10 min after shock is indeed opioidmediated. Of course, even when moderate doses of naltrexone are employed, naltrexone reversibility remains a necessary, but not a sufficient, condition for imputing opioid mediation (Sawynok et al., 1979). Consequently, it is important to demonstrate that other manipulations that undermine opioid hypoalgesia have a similar impact. The next experiment seeks such evidence.

\section{EXPERIMENT 2 \\ Impact of Morphine Tolerance}

In this experiment, we assess the impact of another manipulation that has traditionally been used to infer opioid mediation: morphine tolerance. Unfortunately, our initial attempt to conduct this experiment revealed a basic problem: In the absence of shock, subjects who were morphine-tolerant exhibited a small, but significant, hyperalgesia. This poses a problem, since it could be argued that morphine tolerance attenuates mild shockinduced hypoalgesia simply because it lowers baseline tailflick latencies. There are two ways in which this problem can be addressed. First, one can conduct an analysis of covariance that statistically adjusts for the variance attributable to the baseline differences. Second, one can experimentally manipulate the baseline levels of pain reactivity in the saline controls so that they match the baseline levels of pain reactivity in the morphine-tolerant subjects. In the present experiment, both procedures were used to address the problem of tolerance-induced hyperalgesia.

\section{Method}

Subjects. The subjects were 48 rats of the same age, sex, and strain as were used in the previous experiment.

Apparatus. The apparatus was the same as was used in Experiment 1 .

Procedure. Sixteen subjects received subcutaneous injections of morphine sulphate $(12.5 \mathrm{mg} / \mathrm{kg})$ for 13 days. We have previously shown that this injection regime induces significant tolerance and attenuates another example of shock-induced hypoalgesia that appears to be opioid-mediated (Drugan, Grau, Maier, Madden, \& Barchas, 1981). The other 32 subjects received saline injections. Every day, in the late afternoon, each subject received one injection. Testing was conducted $24 \mathrm{~h}$ after the last injection. Half of the salinetreated subjects and all of the morphine-treated subjects were tested, as described in Experiment 1. Thus, after the subjects had acclimated themselves to the tubes for $15 \mathrm{~min}$, four tailflick tests were conducted to determine baseline levels of pain reactivity. Half of the subjects in each drug condition then received three $0.75-\mathrm{sec}$, 1.0-mA shocks, as described in Experiment 1. The remaining subjects were treated the same, except shock was withheld. Five tailflick tests were then conducted at 2 -min intervals. The remaining saline-treated subjects were treated the same, except that the tailflick device was set to a hotter setting. This was done in an attempt to equate their baseline levels of pain reactivity to the baselines obtained from the morphine-treated subjects.

Statistics. The data were analyzed in the same fashion as described in Experiment 1. In addition, an analysis of covariance (ANCOVA) was performed on the mean levels of pain reactivity observed 6-10 min after shock, or an equivalent period of restraint, in the saline- and morphine-tolerant subjects tested with the tailflick device 
set to the same cool setting. The mean baseline levels of pain reactivity served as the covariate.

\section{Results and Discussion}

The baseline levels of pain reactivity are depicted in Figure 2. It is clear that morphine-tolerant subjects were hyperalgesic compared with the saline controls tested with the tailflick device set to the same cool setting. It is also apparent that saline-treated subjects tested with the tailflick device set to a hotter setting exhibited baseline latencies similar to that observed in morphine-tolerant subjects. An ANOVA confirmed that there was a significant difference between the three conditions $[F(2,45)=12.50$, $p<.005]$. Post hoc comparisons revealed that the subjects in the saline-treated groups, which were tested with the tailflick device adjusted to a cooler setting, exhibited significantly longer tailflick latencies than did all other groups. No other differences were significant.

The impact of morphine tolerance on mild shockinduced hypoalgesia is depicted in Figure 3. It is clear that both of the saline-treated groups were hypoalgesic for the entire 10 min of testing. By contrast, the morphinetolerant subjects appeared hypoalgesic at $2 \mathrm{~min}$ after shock but not at 6-10 min after shock.

An analysis of the data obtained at the 2-min test point revealed a significant shock effect $[F(1,42)=43.49$, $p<.001]$. In addition, there was a significant difference $[F(2,42)=11.44, p<.001]$ in the overall levels of pain reactivity observed across the three test conditions (salinecool, saline-hot, and morphine-tolerant). Importantly, the interaction term revealed that the magnitude of shockinduced hypoalgesia did not depend upon test condition $[F(2,42)=2.54, p>.05]$. Post hoc comparisons showed that the saline-treated shocked subjects tested under the cool setting were hypoalgesic relative to all other groups. Shocked saline-treated and morphine-tolerant subjects tested under the hot setting were hypoalgesic relative to the similarly treated unshocked controls. No other differences were significant.

An analysis of the mean levels of pain reactivity observed 6-10 min after shock revealed that shock had a

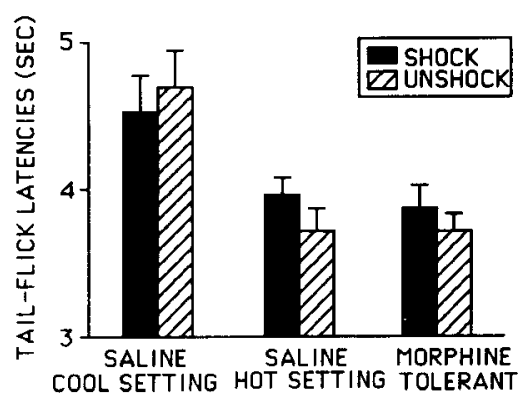

Figure 2. Baseline levels of pain reactivity in morphine-tolerant and saline-treated controls. The saline-treated subjects were tested with the taillick device set to either a "hot" setting or the same "cool" setting used to test the morphine-tolerant subjects. Subjects either were subsequently shocked or served as the unshocked controls. (The error bars indicate the SEM.)

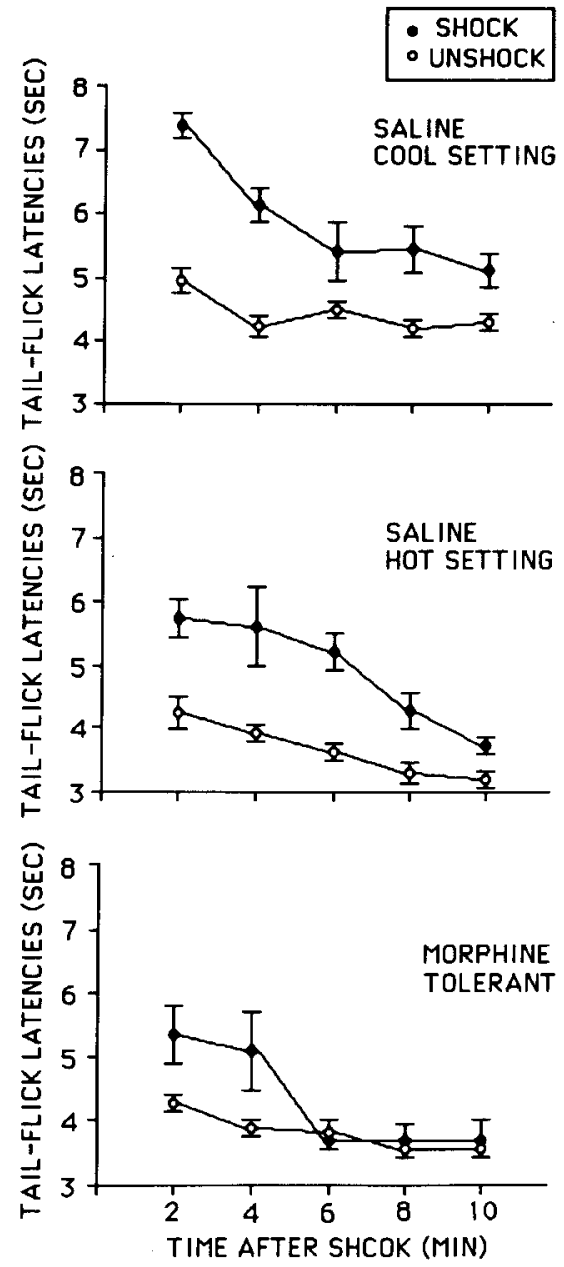

Figure 3. The impact of morphine tolerance on mild shock-induced hypoalgesia. The saline-treated groups depicted in the top panel were tested with the tailnick device set to the same cool setting used to test the morphine-tolerant subjects. The saline-treated groups depicted in the middle panel were tested with the tailflick device set to a hot setting. (The error bars indicate the SEM.)

significant impact $[F(1,42)=15.41, p<.001]$. Again, there was a significant difference $[F(2,42)=16.19$, $p<.001]$ in the levels of pain reactivity observed across the three test conditions (saline-cool, saline-hot, and morphine-tolerant). Importantly, the interaction term revealed that the magnitude of shock-induced hypoalgesia depended upon test condition $[F(2,42)=3.23, p<.05]$. Post hoc comparisons showed that the saline-treated shocked subjects tested under the cool setting were hypoalgesic relative to all other groups. The saline-treated shocked subjects tested under the hot setting were hypoalgesic relative to both the shocked and unshocked morphine-tolerant subjects, as well as to the unshocked saline-treated subjects tested with the tailflick device set to the hot setting. In addition, unshocked saline-treated subjects tested with the tailflick device adjusted to the cool setting exhibited significantly higher latencies than did the 
two morphine-tolerant groups and the unshocked salinetreated group tested under the hot setting. No other differences were significant.

As noted earlier, one could argue that morphine tolerance attenuates the hypoalgesia observed 6-10 min after shock simply because it lowers baseline tailflick latencies. The fact that hypoalgesia was observed 6-10 min after shock in the saline controls that had baseline scores experimentally equated to those of morphine-tolerant subjects suggests this is not the case. An analysis of covariance (ANCOVA) reinforced this conclusion. This analysis was performed on the mean levels of pain reactivity observed 6-10 min after shock in morphine-tolerant subjects and in the saline controls tested with the tailflick device set to the same cool temperature (saline-cool). The analysis revealed a significant effect of both shock $[F(1,27)$ $=8.83, p<.01]$ and drug treatment $[F(1,27)=6.08$, $p<.05]$. Most importantly, the interaction term confirmed that the effect of shock depended upon drug treatment $[F(1,27)=6.18, p<.05]$. Post hoc comparisons of the adjusted means revealed that the saline-treated shocked subjects were hypoalgesic compared with all other groups. No other differences were significant.

Thus, morphine tolerance induced a significant hyperalgesia. In addition, it fully blocked the hypoalgesia observed 6-10 min after shock. Importantly, we demonstrated that this effect cannot be attributed to the effect of morphine tolerance on baseline levels of pain reactivity. Two observations support this claim. First, morphine tolerance attenuated the hypoalgesia observed 6-10 min after shock relative to saline controls that had approximately the same baseline scores. Second, an analysis of covariance revealed that morphine tolerance had a significant effect on the hypoalgesia observed 6-10 min after shock when the variance attributable to the baseline differences was covaried out. These results provide further evidence that the hypoalgesia observed 6-10 min after shock is opioid-mediated. By contrast, morphine tolerance had no impact on the hypoalgesia observed $2 \mathrm{~min}$ after shock, which suggests it is mediated by nonopioid systems.

\section{GENERAL DISCUSSION}

The present experiments explored the role of endogenous opioids in mild shock-induced hypoalgesia. Experiment 1 showed that a low dose of naltrexone blocks the hypoalgesia observed 6-10 min after shock. Experiment 2 demonstrated that this hypoalgesia is also blocked by morphine tolerance. These findings provide strong evidence that the hypoalgesia observed 6-10 min after shock is opioid mediated. In contrast, the hypoalgesia observed 2 min after shock was not affected by either naltrexone (Experiment 1) or morphine tolerance (Experiment 2). This suggests that mild shock also elicits a transient nonopioid hypoalgesia.

Although we have consistently observed both a transient nonopioid and long-lasting opioid hypoalgesia after mild shock, other researchers have failed to observe this pattern of results. For example, Matzel and Miller (1989) tested the impact of a low dose of naloxone $(2 \mathrm{mg} / \mathrm{kg}$ ) on the hypoalgesia observed 0,10 , and $90 \mathrm{sec}$ after a single 1-sec, 4.0-mA tailshock. In contrast to our studies, these authors failed to observe any evidence of a transient nonopioid hypoalgesia. Regarding this failure, a number of points should be noted. First, these authors used much more intense shocks (4.0 mA). Evidence from our laboratory suggests that such an intense tailshock can elicit antinociception in spinalized rats. Thus, unlike our mild shock-induced hypoalgesia, the hypoalgesia observed by Matzel and Miller (1989) may have been mediated by neural systems below the level of the forebrain. Moreover, even if the hypoalgesia observed by Matzel and Miller does depend upon forebrain systems, one would not expect to observe a nonopioid hypoalgesia in their paradigm. The reason for this is that their test procedure, unlike our own, requires removing the subjects from the shock apparatus to test pain reactivity. As Grau (1987b) previously noted, this type of test procedure effectively exposes subjects to a variety of distracting stimuli, which, according to his model (Grau, 1986, 1987b), should act to eliminate the nonopioid hypoalgesia and replace it with one that is opioid-mediated. Elsewhere, Grau (1987b) showed that a postshock distractor has exactly this impact. Thus, Matzel and Miller may have failed to observe a nonopioid hypoalgesia because they distracted their subjects after they were shocked. Supporting this conjecture, other researchers who use a test procedure similar to our own have reported that the hypoalgesia observed immediately after a mild shock ( $15 \mathrm{sec}, 0.9 \mathrm{~mA})$ is not affected by either naloxone or morphine tolerance (Chance \& Rosecrans, 1979a, 1979b).

These observations underscore a point made in the introduction, which is that considerable care needs to be taken when one attempts to generalize across "brief" shock paradigms, for what one defines as brief or mild varies considerably across laboratories. Moreover, even when subjects experience the same amount of shock, the form and the magnitude of the subsequent hypoalgesia can vary depending upon a variety of variables (e.g., whether or not subjects are tested in the shock context [Fanselow, 1984; Maier, 1989], whether or not subjects are distracted [Grau, 1986, 1987a, 1987b], and whether or not the shock is perceived as controllable or uncontrollable [Maier, Drugan, \& Grau, 1982]). Further research is needed to delineate how these variables influence hypoalgesia and the neural mechanisms that mediate changes in pain reactivity.

\section{REFERENCES}

Chance, W. T., * Rosecrans, J. A. (1979a). Lack of cross-tolerance between morphine and autoanalgesia. Pharmacology, Biochemistry \& Behavior, 11, 639-642.

Chance, W. T., Rosecrans, J. A. (1979b). Lack of effect of naloxone on autoanalgesia. Pharmacology, Biochemistry \& Behavior, 11, 643-646.

Drugan, R. C., Grau, J. W., Maier, S. F., Madden, J., \& BarCHAS, J. D. (1981). Cross-tolerance between morphine and the longterm reaction to inescapable shock. Pharmacology, Biochemistry \& Behavior, 14, 677-682 
Drugan, R. C., Mcintyke, T. D., Alpern, H. P., \& Maier, S. F. (1985). Coping and seizure susceptibility: Control over shock protects against bicuculline-induced seizures. Brain Research, 342, 9-17.

Drugan, R. C., Ryan, S. M., Minor, T. R., \& Maier, S. F. (1984). Librium prevents the analgesia and shuttlebox escape deficit typically observed following inescapable shock. Pharmacology, Biochemistry \& Behavior, 21, 749-754.

FANSELOW, M. S. (1984). Shock-induced analgesia on the formalin test: Effects of shock severity, naloxone, hypophysectomy, and associative variables. Behavioral Neuroscience, 98, 79-95.

GraU, J. W. (1984). The influence of naloxone on shock-induced freezing and analgesia. Behavioral Neuroscience, 98, 278-292.

GraU, J. W. (1986). The central representation of an aversive event maintains the opioid and nonopioid forms of analgesia (Doctoral dissertation, University of Pennsylvania, 1985). Dissertation Abstracts International, 46, 4434B.

GraU, J. W. (1987a). Activation of the opioid and nonopioid analgesic systems: Evidence for a memory hypothesis and against the coulometric hypothesis. Joumal of Experimental Psychology: Animal Behavior Processes, 13, 215-225.

GRAU, J. W. (1987b). The central representation of an aversive event maintains the opioid and nonopioid forms of analgesia. Behavioral Neuroscience, 101, 272-288.

Grau, J. W., Illich, P. A., Chen, P.-S., \& Meagher, M. W. (1991). The role of cholinergic systems in pain modulation I: The impact of scopolamine on environmentally-induced hypoalgesia and pain reactivity. Behavioral Neuroscience, 105, 105-124.

MAIER, S. F. (1989). Determinants of the nature of environmentallyinduced hypoalgesia. Behavioral Neuroscience, 103, 131-143.

Maier, S. F., Drugan, R. C., \& Grau, J. W. (1982). Controllability, coping behavior, and stress-induced analgesia in the rat. Pain, 12, 47-56.

Maier, S. F., Ryan, S. M., \& Kurtz, R. (1984). The formalin test and the opioid nature of stress-induced analgesia. Behavioral \& Neural Biology, 41, 54-62.
Matzel, L. D., \& Miller, R. R. (1989). Development of shock-induced analgesia: A search for hyperalgesia. Behavioral Neuroscience, 103, 850-856.

Meagher, M. W., Grau, J. W., \& King, R. A. (1989). Frontal cortex lesions block the opioid and nonopioid hypoalgesia elicited by brief shocks but not the nonopioid hypoalgesia elicited by long shocks. Behavioral Neuroscience, 103, 1366-1371.

Meagher, M. W., Grau, J. W., \& King, R. A. (1990). The role of supraspinal systems in environmentally induced antinociception: The effects of spinalization and decerebration on brief and long shockinduced antinociception. Behavioral Neuroscience, 104, 328-338.

Sawynok, J., Pinsky, C., \&aBella, F. S. (1979). Minireview on the specificity of naloxone as an opiate antagonist. Life Sciences, 25, 1621-1632.

Terman, G. W., Lewis, J. W., \& Liebeskind, J. C. (1986). Two opioid forms of stress analgesia: Studies of tolerance and cross tolerance. Brain Research, 368, 101-106.

Terman, G. W., Shavit, Y., Lewis, J. W., Cannon, J. T., LIEBESKIND, J. C. (1984). Intrinsic mechanisms of pain inhibition: Activation by stress. Science, 226, 1270-1277.

Watkins, L. R., Cobelli, D. A., Faris, P., Aceto, M. D., Mayer, D. J. (1982). Opioid vs non-opiate footshock-induced analgesia (FSIA): The body region shocked is a critical factor. Brain Research, 242, 299-308.

Watkins, L. R., Kinscheck, I. B. , MAYER, D. J. (1983). The neural basis of footshock analgesia: The effect of periaqueductal gray lesions and decerebration. Brain Research, 276, 317-324.

WATKINS, L. R., \& MAYER, D. J. (1982). Organization of endogenous opiate and nonopiate pain control systems. Science, 216, 1185-1192.

(Manuscript received February 16, 1990; revision accepted for publication September 27, 1990.) 